\title{
The Impact of Alpha-Lipoic Acid on Insulin Resistance and Inflammatory Parameters in Patients with Type 2 Diabetes Mellitus and Cardiac Autonomic Neuropathy
}

\author{
Victoria Serhiyenko ${ }^{1, *}$, Marta Hotsko ${ }^{1}$, Alexandr Serhiyenko ${ }^{1}$, Oksana Snitynska ${ }^{1}$, \\ Ludmila Serhiyenko ${ }^{2}$, Volodymyr Segin ${ }^{3}$ \\ ${ }^{1}$ Department of Endocrinology, Danylo Halytsky Lviv National Medical University, Lviv, Ukraine \\ ${ }^{2}$ Department of Medical Biology, Danylo Halytsky Lviv National Medical University, Lviv, Ukraine \\ ${ }^{3}$ Lviv Regional State Clinical Treatment and Diagnostic Endocrinology Center, Lviv, Ukraine
}

Email address:

serhiyenkov@gmail.com (V. Serhiyenko), martahotsko@ukr.net (M. Hotsko), serhiyenkoa@gmail.com (A. Serhiyenko), snitja@mail.ru (O. Snitynska), serhiyenkoludmila@gmail.com (L. Serhiyenko), volodymyr.segin@gmail.com (V. Segin)

${ }^{*}$ Corresponding author

\section{To cite this article:}

Victoria Serhiyenko, Marta Hotsko, Alexandr Serhiyenko, Oksana Snitynska, Ludmila Serhiyenko, Volodymyr Segin. The Impact of AlphaLipoic Acid on Insulin Resistance and Inflammatory Parameters in Patients with Type 2 Diabetes Mellitus and Cardiac Autonomic Neuropathy. American Journal of Internal Medicine. Vol. 8, No. 5, 2020, pp. 197-203. doi: 10.11648/j.ajim.20200805.11

Received: July 7, 2020; Accepted: July 22, 2020; Published: August 13, 2020

\begin{abstract}
Development of chronic complications in type 2 diabetes mellitus (T2DM), namely cardiac automonic neuropathy (CAN), had been implicated as an independent risk factor for cardiovascular mortality. This paper presents the results obtained during the study of possible alpha-lipoic acid (ALA) impact on the state of insulin resistance (IR), the content of some pro- and anti-inflammatory factors in patients with T2DM and definite stage of CAN. Our study involved 33 persons with diagnosed T2DM and definite CAN, which were allocated to one of two groups. The control group (15 patients) received standard hypoglycemic treatment, whereas the intervention group (18 patients) received ALA $600 \mathrm{mg}$ in film-coated tablets/q.d. in addition to standard therapy. Treatment period was 3 months in duration. Levels of glycated hemoglobin A1c, glucose, immunoreactive insulin (IRI), leptin, interleukin (IL) IL-6 and IL-8, high sensitivity C-reactive protein (hs-CRP), tumor necrosis factor-alpha (TNF-alpha) in the blood were measured. TNF-alpha/IL-10 ratio and Homeostasis model assessment IR (HOMA-IR) were calculated. The significant reduction in glucose, IRI, leptin concentration and HOMA-IR parameters; decrease in the pro-inflammatory link activity, namely hs-CRP, TNF-alpha, IL-6 and IL-8 levels, TNF-alpha/IL-10 ratio, with no changes in IL-10 content after 3 months of treatment was found. The results of our study demonstrated the decrease in the pro-inflammatory link activity after treatment and allow us to consider ALA as one of the promising drug for complex treatment of definite stage of CAN in patients with T2DM.
\end{abstract}

Keywords: Diabetic Cardiac Autonomic Neuropathy, Alpha-Lipoic Acid, Inflammation, Type 2 Diabetes Mellitus

\section{Introduction}

Cardiac autonomic neuropathy (CAN) is one of the most frequent type of autonomic neuropathy, that was defined by the Toronto consensus panel as the impairment of cardiovascular autonomic control among patients with diagnosed diabetes mellitus (DM) following the exclusion of other causes $[1,2]$. Diabetic CAN is an independent risk factor for major cardiovascular events, development of heart arrhythmias, silent myocardial ischaemia, sudden cardiac death and cardiovascular mortality. Development of CAN in type $2 \mathrm{DM}$ (T2DM) is associated with resting tachycardia, decreased tolerance to the physical loadings, orthostatic hypotension $(\mathrm{OH})$, "silent" myocardial infarction, long QT and sudden arrhythmic death syndrome [1, 3-7].

Therefore, screening for CAN is important for predicting the prognosis of patients with DM and formulation of new effective treatment strategy is extremely important too. The impetus for this work was prompted by the hypothesis that 
physiological antioxidants, including alpha-lipoic acid (ALA), a powerful lipophilic "trap" of free radicals, promote the prevention of neurovascular disorders, provide the basis for the pathogenetically-based application of ALA in the treatment of diabetic angio-, neuropathy, in particular including diabetic CAN.

The results of Deutsche Kardiale Autonome Neurophathie (DEKAN) study demonstrated that treatment with ALA, using a well-tolerated dose of $800 \mathrm{mg}$ /day orally during four months was associated with improvement of heart rate variability (HRV) parameters [8,9]. Despite the data that ALA seems to be promising drug to improve HRV, neuropathic symptoms, trials aimed to evaluate features of ALA among patients with DM without diagnosed chronic heart diseases are few, and obtained results do not confirm its effectiveness [9, 10]. The effects of ALA upon endothelial function and markers of oxidative stress (OS) were age dependent. In contrast, a review of many clinical trials using chronic antioxidant therapy was not able to demonstrate the benefits for cardiovascular diseases [10].

This study focused on determining whether ALA has a potential impact on inflammation, glycaemic control, insulin resistance (IR) parameters in patients with definite CAN and T2DM.

\section{Materials and Methods}

\subsection{Patient Characteristics}

This study was designed as an open-label comparative controlled study. A total of 33 T2DM patients with definite CAN aged between 50-59 yrs., DM duration between 1-6 yrs. with glycated hemoglobin A1c (HbA1c) level of $7.03 \pm 0.13 \%$ and median body mass index (BMI) $27.1 \pm 0.32 \mathrm{~kg} / \mathrm{m}^{2}$ were enrolled. For purposes of the study patients were allocated to two groups: the control group (15 patients) received standard hypoglycemic treatment, whereas the intrevention group (18 patients) received ALA $600 \mathrm{mg}$ in film-coated tablets/q.d. in addition to standard therapy. Treatment period was 3 months in duration. The age, sex distribution in the two groups was similar, as well as BMI and duration of DM, which made groups homogeneous as possible. Clinical characteristics of patients are presented in Table 1 .

Table 1. Clinical characteristics of patients.

\begin{tabular}{|c|c|c|}
\hline \multirow{3}{*}{ Parameter } & \multicolumn{2}{|c|}{ Patients with T2DM and definite CAN $(n=33)$} \\
\hline & Control (n=15) & Intervention $(\mathrm{n}=18)$ \\
\hline & Group 1 & Group 2 \\
\hline Age (years) & $55.33 \pm 0.95$ & $54.83 \pm 0.87$ \\
\hline \multicolumn{3}{|l|}{ Gender } \\
\hline Male (\%) & $8 / 53.3 \%$ & $10 / 55.6 \%$ \\
\hline Female (\%) & $7 / 46.7 \%$ & $8 / 44.4 \%$ \\
\hline Diabetes duration (years) & $3.6 \pm 0.42$ & $3.5 \pm 0.42$ \\
\hline $\operatorname{BMI}\left(\mathrm{kg} / \mathrm{m}^{2}\right)$ & $28.89 \pm 0.16$ & $28.18 \pm 0.33$ \\
\hline \multicolumn{3}{|l|}{ Medications } \\
\hline ACE inhibitors (\%) & $12 / 80 \%$ & $14 / 77.8 \%$ \\
\hline Beta-blockers (\%) & $3 / 20 \%$ & $2 / 11.11 \%$ \\
\hline Metformin (\%) & $11 / 73.3 \%$ & $10 / 55.6 \%$ \\
\hline Combined hypoglycemic therapy (\%) & $3 / 20 \%$ & $7 / 38.9 \%$ \\
\hline Hypertension (\%) & $12 / 80 \%$ & $15 / 83.3 \%$ \\
\hline
\end{tabular}

Abbreviations: T2DM: type 2 diabetes mellitus, CAN: cardiac autonomic neuropathy, BMI: body mass index, ACE: angiotensin-converting enzyme.

The standard hypoglycemic management of T2DM, which included lifestyle intervention, namely appropriate dietary program, physical activity and oral antihyperglycemic drugs, was performed. The standard treatment of patients in the groups remained unchanged during the period of the study, however to patients from intervention group was prescribed $600 \mathrm{mg}$ of ALA (Thioctic Acid) in film-coated tablets/q.d. for three months.

The inclusion criteria were specified as follows: patients aged between 50 and 60 years with T2DM, archieved optimal and suboptimal glycemic control; definite stage of CAN confirmed by cardiovascular autonomic reflex tests (CART's), diabetic polyneuropathy; consent to maintain lifestyle intervention, namely appropriate dietary program and physical activity. Exclusion criteria were specified as follows: uncontrolled DM, ketosis, distal neuropathy due to medical conditions other than diabetes (pharmalogical agents, chronic alcoholism, neurological disease), ischemic form of diabetic foot, peripheral vascular diseases, history of acute pancreatitis, hypothyroidism, neoplasms, women during lactation and pregnancy, T1DM patients, hypersensitivity to ALA. Treatment with anticonvulsants, antidepressants, neuroleptic agents, cytostatic agents, opiates, capsaicin, B group vitamins, gamma-linoleic acid, aldose reductase inhibitors, warfarin, isotretinoin (accutane), antioxidants (including ALA medications) in the six months preceding to the inclusion to the study were also exclusion criteria. Persons suffering from severe diseases of the lungs, liver, heart, pancreas and intestines were also excluded from the study. 
The study was conducted for 2 years. All patients were examined before the start of the trial and after the end of the treatment period. All involved patients did not take ALA and was on stable regime of hypoglycemic and antihypertensive treatment for 6 months.

\subsection{Primary and Additional Study Outcomes}

The positive effect of ALA, especially a significant decrease of the glucose, immunoreactive insulin (IRI) concentrations, Homeostasis Model Assessment (HOMA) IR (HOMA-IR) parameters; high-sensitivity C-reactive protein (hs-CRP), leptin, tumour necrosis factor-alpha (TNF-alpha), interleukin (IL) IL-6 and IL-8, TNF-alpha/IL-10 levels, but no significant changes of IL-10 content compared with the control group after 3 month of treatment was confirmed.

\subsection{Methods}

Cardiovascular autonomic reflex tests are considered as the "gold" standard tests in the assessment of CAN. Based on the Subcommittee of the Toronto Consensus Panel on Diabetic Neuropathy, one abnormal result of CART is sufficient for CAN diagnosis, but identifies the possible development of early CAN, that must be confirmed over time, the presence of two or three abnormal test results confirm definite $\mathrm{CAN}$; and additional development of $\mathrm{OH}$ indicate severe stage of complication [11, 12].

Screening for CAN, that included five CART's, timeand frequency-domain HRV tests was performed for all enrolled patients. The obtained results were evaluated as follows: pathological - as " 1 " scores, borderline values as " 0.5 " scores, normal values - as " 0 " score. The individual scores were summed up and the median score for involved patients was $2.8 \pm 0.32$ [11, 12]. Glucose oxidase method was used for measurement of glucose concentration in the blood, while a highly sensitive method of ion-exchange liquid chromatography with D-10 analyzer and BIO-RAD reagents (USA) was used for HbA1c determination.

Serum IRI level was measured by using commercial kits from immunogen insulin immunoradiometric assay reagents (Czech Republic); IL-6, IL-8 and IL-10, TNFalpha - from Vector-Best (Russia); leptin - from Immunotech Leptin (Czech Republic) test kits; hs-CRPfrom diagnosis-related group (USA). TNF-alpha/IL-10 ratio was calculated. For the calculation of HOMA-IR was used formula: fasting IRI $(\mathrm{mcIU} / \mathrm{mL}) \mathrm{x}$ fasting glucose $(\mathrm{mmol} / \mathrm{L}) / 22.5$ [13].

\subsection{Statistical Analysis}

To compare repeated measurements (before and after treatment) the Wilcoxon signed-rank test was used. A comparison of the obtained data in the two groups was carried out using the nonparametric Mann-Whitney test. All data are expressed as median (Me) and interquartile range (Q25-Q75). Statistical significance was set at $\mathrm{p}<0.05$. All tests were performed using the ANOVA (MicroCal Origin v. 8.0) software.

\section{Results}

We found out that HbA1c of patients with T2DM and definite CAN was not statistically significantly influenced by the treatment $(p>0.05)$. There were no differences between the groups in levels of preprandial blood glucose, IRI concentrations and HOMA-IR parameters at baseline. Baseline and post-treatment parameters of blood glucose, IRI concentrations and HOMA-IR parameters in patients with T2DM and definite stage of CAN are shown in the Table 2.

Table 2. Baseline and post-treatment parameters of blood glucose, IRI concentrations and HOMA-IR in patients with T2DM and definite stage of CAN after 3 months of ALA therapy.

\begin{tabular}{|c|c|c|c|c|c|c|}
\hline \multirow{4}{*}{ Parameter } & \multicolumn{6}{|c|}{ Patients with T2DM and definite CAN $(n=33)$} \\
\hline & \multicolumn{2}{|l|}{ Control group } & \multicolumn{2}{|l|}{ Intervention group } & \multirow{2}{*}{$\begin{array}{l}\text { Control group } \\
\mathrm{n}=15\end{array}$} & \multirow{2}{*}{$\begin{array}{l}\text { Intervention group } \\
\mathrm{n}=18 \\
\end{array}$} \\
\hline & $\mathrm{n}=15$ & & $\mathrm{n}=\mathbf{1 8}$ & & & \\
\hline & Baseline & After treatment & Baseline & After treatment & After treatment & After treatment \\
\hline Fasting glucose $(\mathrm{mmol} / \mathrm{L})$ & $7.3[6.1 ; 8.0]$ & $7.4[5.9 ; 7.8]$ & $6.4[6.1 ; 7.3]$ & $5.9[5.2 ; 6.4]^{*}$ & $7.4[5.9 ; 7.8]$ & $5.9[5.2 ; 6.4]^{\#}$ \\
\hline Fasting IRI (mcIU/mL) & $29.4[22.4 ; 34.2]$ & $28.0[21.1 ; 33.9]$ & $27.65[24.3 ; 30.2]$ & $21.35[19.7 ; 24.0]^{* *}$ & $28.0[21.1 ; 33.9]$ & $21.35[19.7 ; 24.0]^{\#}$ \\
\hline HOMA-IR & $9.8[6.07 ; 12.16]$ & $9.3[5.8 ; 11.4]$ & $8.03[6.19 ; 9.28]$ & $5.28[4.83 ; 6.48]^{* *}$ & $9.3[5.8 ; 11.4]$ & $5.28[4.83 ; 6.48]^{\# \#}$ \\
\hline
\end{tabular}

Abbreviations: T2DM type 2 diabetes mellitus, CAN cardiac autonomic neuropathy, IRI immunoreactive insulin, IR insulin resistance, HOMA-IR Homeostasis model assessment IR.

The results are presented as median $(\mathrm{Me})$ and interquartile range (Q25-Q75). ${ }^{*} \mathrm{p}<0.05$ compared to baseline, $* * \mathrm{p}<0.01$ compared to baseline, $\# \mathrm{p}<0.05$ compared to data of control group, obtained after treatment. \#\# $\mathrm{p}<0.01$ compared to data of control group, obtained after treatment.

As shown in the Table 2 the outcome of the intervention group was better than that of the control group. After 3 months of treatment with ALA levels of preprandial glycemia $(p<0.05)$, IRI $(p<0.01)$ and HOMA-IR parameters $(p<0.01)$ were lower compared to pretreatment values. Compared to the data, obtained in the control group, more pronounced decrease in fasting glucose $(p<0.05)$, IRI $(p<0.05)$ and
HOMA-IR parameters $(p<0.01)$ was found. Investigated parameters did not differ between baseline levels and after three months in the control group.

Baseline and post-treatment parameters of hs-CRP, leptin, TNF-alpha, IL-6, IL-8, IL-10 concentrations and TNFalpha/IL-10 ratio in patients with T2DM and definite stage of CAN are given in the Table 3 . 
Table 3. Baseline and post-treatment parameters of hs-CRP, leptin, TNF-alpha, IL-6, IL-8, IL-10 concentrations and TNF-alpha/IL-10 ratio in patients with T2DM and definite stage of CAN after 3 months of ALA therapy.

\begin{tabular}{|c|c|c|c|c|c|c|}
\hline \multirow{4}{*}{ Parameter } & \multicolumn{6}{|c|}{ Patients with T2DM and definite CAN (n=33) } \\
\hline & \multicolumn{2}{|l|}{ Control group } & \multicolumn{2}{|l|}{ Intervention group } & \multirow{2}{*}{$\begin{array}{l}\text { Control group } \\
\mathrm{n}=15 \\
\end{array}$} & \multirow{3}{*}{$\begin{array}{l}\text { Intervention group } \\
\mathrm{n}=18 \\
\text { After treatment } \\
\end{array}$} \\
\hline & $\mathrm{n}=\mathbf{1 5}$ & & $\mathrm{n}=\mathbf{1 8}$ & & & \\
\hline & Baseline & After treatment & Baseline & After treatment & After treatment & \\
\hline hs-CF & $2.48[1.53 ; 4.43]$ & $2.25[1.5 ; 4.33]$ & $2.47[2.15 ; 3.98]$ & $2.19[1.97 ; 3.04]^{*}$ & $2.25[1.5 ; 4.33]$ & $2.19[1.97 ; 3.04]$ \\
\hline Leptin & 22.1 & 23.7] & 24.8] & $15.55[13.6 ; 19.7]^{*}$ & 23.7] & $15.55[13.6 ; 19.7]^{\#}$ \\
\hline TNF-alpha (pg/mL) & $5.9[4.4 ; 7.1]$ & $5.7[4.6 ; 7.3]$ & $5.85[5.1 ; 6.2]$ & $4.9[4.5 ; 5.2]^{* * *}$ & $5.7[4.6 ; 7.3]$ & $4.9[4.5 ; 5.2]^{\#}$ \\
\hline IL-6 (pg/mL) & $5.36[4.31 ; 6.15]$ & $5.28[4.74 ; 6.08]$ & $5.87[4.19 ; 7.28]$ & $4.33[3.54 ; 5.44]^{*}$ & $5.28[4.74 ; 6.08]$ & $4.33[3.54 ; 5.44]^{\#}$ \\
\hline IL-8 (pg/mL) & $6.9[5.5 ; 9.2]$ & $6.7[5.6 ; 8.7]$ & $6.25[5.7 ; 7.2]$ & $5.25[4.7 ; 5.7]^{*}$ & $6.7[5.6 ; 8.7]$ & $5.25[4.7 ; 5.7]^{\#}$ \\
\hline IL-10 (pg/mL) & $13.5[9.3 ; 16.4]$ & $11.7[10.1 ; 17.7]$ & $13.45[10.4 ; 16.8]$ & $15.4[12.5 ; 18.6]$ & $11.7[10.1 ; 17.7]$ & $15.4[12.5 ; 18.6]$ \\
\hline TNF-alpha/IL-10 (\%) & $37.9[26.4 ; 52.7]$ & $41.6[28.8 ; 58.7]$ & $40.95[35.1 ; 51.2]$ & $32.55[27.1 ; 39.8]^{* *}$ & $41.6[28.8 ; 58.7]$ & $32.55[27.1 ; 39.8]^{\#}$ \\
\hline
\end{tabular}

Abbreviations: T2DM type 2 diabetes mellitus, CAN cardiac autonomic neuropathy, hs-CRP high-sensitivity C-reactive protein, TNF-alpha tumour necrosis factor-alpha, IL interleukin, TNF-alpha/IL-10 tumour necrosis factor-alpha/intrerleukin-10 ratio.

The results are presented as median (Me) and interquartile range (Q25-Q75). ${ }^{*} \mathrm{p}<0.05$ compared to baseline, ${ }^{* *} \mathrm{p}<0.01$ compared to baseline, ${ }^{\#} \mathrm{p}<0.05$ compared to data of control group, obtained after treatment

There were no differences between the groups in leves of hs-CRP, leptin and TNF-alpha at baseline. As shown in the Table 3 after three months of ALA prescription to patients with $\mathrm{T} 2 \mathrm{DM}$ and definite CAN a statistically significant reduction in TNF-alpha $(p<0.001)$, leptin $(\mathrm{p}<0.05)$ and hsCRP $(p<0.05)$ levels was found. Compared to the posttreatment values, obtained in the control group, more pronounced decrease in TNF-alpha $(\mathrm{p}<0.05)$ and leptin $(p<0.05)$ was found. Investigated parameters did not differ between baseline levels and after 3 months in the control group (Table 3).

Obtained results showed that there were no differences between the groups in leves of IL-6, IL-8, IL-10 concentrations, and TNF-alpha/IL-10 ratio at baseline. After 3 months of treatment content of IL-6 $(p<0.05)$, IL-8 $(p<0.05)$ and TNF-alpha/IL-10 ratio $(\mathrm{p}<0.01)$ was statistically significant lower compared with the pretreatment values and values, obtained in control group, namely IL- $6(\mathrm{p}<0.05)$, IL-8 $(\mathrm{p}<0.05)$ and TNF-alpha/IL-10 ratio $(\mathrm{p}<0.05)$. In control group, no positive dynamic of the concentrations of studied parameters was found $(p>0.05)$. IL-10 levels did not differ between two groups before and after treatment, showing no changes, compared with pretreatment values, in either group (Table 3).

During the period of clinical research, good tolerability and safety of ALA were established, which are confirmed by the absence of serious and unpredictable side effects.

\section{Discussion}

Alpha-lipoic acid has been considered as a powerful micronutrient presenting a range of pharmacological properties, antioxidant capacity, ability to regenerate other antioxidants such as vitamins $\mathrm{E}$ and $\mathrm{C}$, in addition to enhance the effects of superoxide dismutase, coenzyme Q 10 and glutathione (GSH), reduce and/or reverse age-related GSH redox decline [14-16]. The following explanations have been proposed to explain beneficial effects of ALA in reducing the age-associated alterations in GSH: 1. Impossible delivery of exogenous GSH to tissues such as the brain and heart. 2. The bioavailability of cysteine delivery agents (e.g., $\mathrm{N}$-acetylcysteine) is low. However,
ALA is effective agent to restore both the age-associated decline in thiol redox ratio as well as increase of cerebral GSH levels that otherwise decline with age, as it is easily taken up into neural tissues $[17,18]$.

Alpha-lipoic acid exibit direct free radical scavenging properties both in vitro and in vivo. There is a strong link between hyperglycaemia, OS, increased production and decreased clearance of reactive oxygen species (ROS), low grade inflammation and development/progression of T2DM and its complications. Oxidative stress has been suggested to contribute to defective nerve blood supply and endoneurial oxidative damage $[16,19]$. Some studies have demonstrated the ability of ALA to reduce expression of vascular cell adhesion molecule-1 (VCAM-1) and endothelial adhesion of human monocytes in vitro. Futhermore, ALA prevents the upregulation of intercellular adhesion molecule-1 and VCAM-1 in spinal cords and TNF-alpha stimulated cultured brain endothelial cells [20,21].

Alpha-lipoic acid is largely known as an anti-inflammatory and antioxidant molecule, the effects of which are exerted through the modulation of nuclear factor kappa-light chainenhancer of activated B cells (NF-kB). Different inflammatory cytokines, including IL- $1 \beta$ and IL-6, in different cell types and tissues, are modulated by NF-kB. IL$1 \beta$ and IL-6 undergo DNA methylation-dependent modulation in neural models and pave the road to study of the epigenetic mechanisms triggered by ALA [19].

Alpha-lipoic acid may effectively reduce OS by preventing lipid peroxidation (LPO) and protein damage as other antioxidants significantly inhibit the ROS such as proxy nitrite, nitric oxide, hydroxyl radicals, superoxide anion in the membrane and aquatic environment $[14,16]$.

Chronic hyperglycemia is associated with OS and the existence of other trigger factors are supposed to phosphorylate the nuclear factor of kappa light polypeptide gene enhancer in B-cells inhibitor (IkB) resulting in its degradation and NF-kB activation. Phosphorylation and degradation of $\mathrm{IkB}$ are considered as a key step for the regulation of NF-kB complexes, which induces the transcription of several molecules related to the migration of monocytes, vascular adhesion and inflammation. The 
inhibition of NF-kB by ALA is probably related to its possibility to decrease I $\mathrm{B}$ degradation through modulation of upstream kinases like mitogen-activated protein kinase or to inhibit protein kinase $\mathrm{C}$ which is also able to phosphorylate IkB [10].

Alpha-lipoic acid has drawn considerable attention due to its properties to prevent/treat chronic complications of DM [14]. ALA prescription is an effective strategy for mitochondrial protection, improvement of mitochondrial function and structure, inhibition of the age-associated increase of oxidative damage, partial restoration of the key enzymes activity, increase of ATP production, especially in the state of chronic inflammation [10, 22-25].

Some studies showed that treatment with ALA either i.v. or p.o. was associated with glucose disposal improvement in patients with T2DM [26]. Also, some other studies findings suggest improvement in HbA1c, LPO, antioxidant enzymes and inflammatory markers. ALA as a compound with strong antioxidant potential, has a function in decreasing damages caused by chronic low-grade inflammation and diabetic neuropathies [15, 27-29].

The results of Irbesartan and Lipoic Acid in Endothelial Dysfunction (ISLAND) clinical trial showed that supplementation with ALA in daily dose of $300 \mathrm{mg}$ for $4 \mathrm{wks}$ was associated with the $15 \%$ significant decrease in serum IL-6 levels. As IL-6 is a recognized marker of inflammation in coronary atherosclerotic plaques, and regulates the expression of other inflammatory cytokines, such as TNFalpha and IL-1, this finding may prove the anti-inflammatory effects of ALA. Nevertheless, the body of evidence is currently too limited and could not be considered as conclusive, so futher investigations are needed [30-32].

Our study suggests that administration of ALA contributed to a statistically significant decrease of glucose, IRI concentrations, HOMA-IR parameters; TNF-alpha, hs-CRP, IL-6 and IL-8, leptin levels and TNF-alpha/IL-10 ratio, but does not significantly affect the content of IL-10 content in patients with T2DM and definite CAN. The obtained data may indicate a decrease in the activity of the proinflammatory link of the immune response and allow us to consider ALA as a promising agent in the CAN complex treatment in patients with T2DM. Our study has several limitations. First, the sample size was small, second - short duration. Finally, obtained results must be confirmed and clarified in futher studies.

\section{Conclusion}

Numerous results of experimental and clinical trials suggest unique properties of ALA, namely its antioxidant effects, restoration of vitamins levels (such as vitamin $\mathrm{E}$ and $\mathrm{C}$ ), inhibition of glycation reactions, prevention of beta-cell destruction, improvement of neurons function and conduction.

ALA has a valuable therapeutic option in prevention and treatment of chronic complications of DM, mainly due to its essential role in mitochondrial bioenergetic reactions. Our study suggests that administration of ALA contributed to a statistically significant decrease of glucose, IRI concentrations, HOMA-IR parameters; TNF-alpha, hs-CRP, leptin, IL-6, IL-8 levels and TNF-alpha/IL-10 ratio, but does not significantly affect the content of IL-10 in patients with T2DM and definite CAN. However, further studies with longer duration and larger population are needed to validate our findings and provide evidence for the hidden therapeutic potential of ALA.

\section{Source of Finance}

During this study, no financial or spiritual support was received neither from any pharmaceutical company that has a direct connection with the research subject, nor from a company that provides or produces medical instruments and materials, which may negatively affect the evaluation process of this study.

\section{Conflict of Interest}

All the authors do not have any possible conflicts of interest.

\section{Human Rights Statement and Informed Consent}

All procedures followed were in accordance with the ethical standards of the Institutional Ethical Committee of the Danylo Halytsky Lviv National Medical University (protocol № 1 from 01 Jan 2016) and Helsinki Declaration (revised 2013). Written informed consent was obtained from all patients before the inclusion in the study. Patients were examined and treated by the endocrinologist at the Department of Endocrinology of Danylo Halytsky Lviv National Medical University based on Lviv Regional State Clinical Treatment and Diagnostical Endocrinological Center. All patients were informed orally about the study and signed the corresponding informed consent prior their inclusion in the study.

\section{Acknowledgements}

We thank all the neurologists and nurses in the Lviv Regional State Clinical Treatment and Diagnostic Endocrinology Center.

\section{References}

[1] Spallone V, Ziegler D, Freeman R, Bernardi L, Frontoni S, Pop-Busui $\mathrm{R}$ et al. Toronto Consensus Panel on Diabetic Neuropathy. Cardiovascular autonomic neuropathy in diabetes: clinical impact, assessment, diagnosis, and management. Diabetes Metab Res Rev. 2011; 27 (7): 639-653. doi: 10.1002/dmrr.1239.

[2] Spallone V. Update on the impact, diagnosis and management of cardiovascular autonomic neuropathy in diabetes: what is defined, what is new, and what is unmet. Diabetes Metab. J. 2019; 43 (1): 3-30. doi: 10.4093/dmj.2018.0259. 
[3] Pop-Busui R, Boulton AJM, Feldman EL, Bril V, Freeman R, Malik RA et al. Diabetic neuropathy: A position statement by the American Diabetes Association. Diabetes Care. 2017; 40: 136-154. doi: $10.2337 / \mathrm{dc} 16-2042$.

[4] Ziegler D, Keller J, Maier C, Pannek J. Diabetic neuropathy. Exp Clin Endocrinol Diabetes. 2014; 122: 406-415. doi: $10.1055 / \mathrm{s}-0034-1366435$.

[5] Tesfaye S, Boulton AJ, Dyck PJ Freeman R, Horowitz M, Kempler P et al. Diabetic neuropathies: update on definitions, diagnostic criteria, estimation of severity, and treatments. Diabetes Care. 2010; 33 (10): 2285-2293. doi: 10.2337/dc101303.

[6] Serhiyenko VA, Serhiyenko AA. Diabetic cardiac autonomic neuropathy: Do we have any treatment perspectives? World J Diabetes. 2015; 6 (2): 245-258. doi: 10.4239/wjd.v6.i2.245.

[7] Serhiyenko VA, Serhiyenko AA. Cardiac autonomic neuropathy: Risk factors, diagnosis and treatment. World J Diabetes. 2018; 9 (1): 1-24. doi: 10.4239/wjd.v9.i1.1.

[8] Ziegler D, Schatz H, Conrad F, Gries FA, Ulrich H, Reichel G. Effects of treatment with the antioxidant alpha-lipoic acid on cardiac autonomic neuropathy in NIDDM patients. A 4-month randomized controlled multicenter trial (DEKAN Study). Diabetes Care. 1999; 20 (2): 369-373. doi: 10.2337/diacare.20.3.369.

[9] Lee SJ, Jeong SJ, Lee YC, Lee YH., Lee JE., Kim CH et al. Effects of high-dose $\alpha$-lipoic acid on heart rate variability of type 2 diabetes mellitus patients with cardiac autonomic neuropathy in Korea. Diabetes Metab J. 2017; 41 (4): 275-283. Doi: 10.4093/dmj.2017.41.4.275.

[10] Gomes MB, Negrato CA. Alpha-lipoic acid as a pleiotropic compound with potential therapeutic use in diabetes and other chronic diseases. Diabetol Metab Syndr. 2014; 6 (1): 80. doi: 10.1186/1758-5996-6-80.

[11] Ewing DJ, Martyn CN, Young RJ, Clarke BF. The value of cardiovascular autonomic function tests: 10 years experience in diabetes. Diabetes Care. 1985; 8 (5): 491-498. doi: 10.2337/diacare.8.5.491.

[12] Spallone V, Bellarvere F, Scionti L, Maule S, Quadri R, Bax G et al. Diabetic Neuropathy Study Group of the Italian Society of Diabetology. Recommendations for the use of cardiovascular tests in diagnosing diabetic autonomic neuropathy. Nutr Metab Cardiovascular Dis. 2011; 21 (1): 6978. doi: $10.1002 /$ dmrr. 1239

[13] Matthews DR, Hosker JP, Rudenski AS, Naylor BA, Treacher DF, Turner RC. Homeostasis model assessment: insulin resistance and beta-cell function from fasting plasma glucose and insulin concentrations in man. Diabetologia.1985; 28 (7): 412-419. doi: 10.1007/BF00280883.

[14] Rochette L, Ghibu S, Muresan A, Vergely C. Alpha-lipoic acid: molecular mechanisms and therapeutic potential in diabetes. Can J Physiol Pharmacol. 2015; 93 (12): 1021-1027. doi: 10.1139/cjpp-2014-0353.

[15] Ahmadvand H, Jamor P. Effects of alpha lipoic acid on level of NO and MPO activity in diabetic rats. Ann Res Antioxid. 2017; 2: e04. http://annresantioxidants.com. Accessed 04 Jun 2017.

[16] Vallianou N, Evangelopoulos A, Koutalas P. Alpha-lipoic acid and diabetic neuropathy. Rev Diabet Stud. 2009; 6 (4): 230-

\section{6. doi: 10.1900/RDS.2009.6.230.}

[17] Suh JH, Wang H, Liu RM, Hagen TM. (R)-alpha-lipoic acid reverses the agerelated loss in GSH redox status in postmitotic tissues: evidence for increased cysteine requirement for GSH synthesis. Arch Biochem Biophys. 2004; 423 (1): 126-135. doi: 10.1016/j.abb.2003.12.020.

[18] Golbidi S, Badran M, Laher I. Diabetes and alpha lipoic acid. Front Pharmacol. 2011; 2: 69. doi: 10.3389/fphar.2011.00069.

[19] Dinicola S, Proietti S, Cucina A, Bizzarri M, Fuso A. Alphalipoic acid downregulates IL-1 $\beta$ and IL-6 by DNA hypermethylation in SK-N-BE neuroblastoma cells. Antioxidants (Basel). 2017; 6 (4): E74. doi: 10.3390/antiox6040074.

[20] Shay K, Hagen TM. Age-associated impairment of Akt phosphorylation in primary rat hepatocytes is remediated by alpha-lipoic acid through PI3 kinase, PTEN, and PP2A. Biogerontology. 2009; 10 (4): 443-456. doi: 10.1007/s10522008-9187-x.

[21] Packer L, Cadenas E. Lipoic acid: energy metabolism and redox regulation of transcription and cell signalling. J Clin Biochem Nutr. 2011; 48 (1): 26-32. doi: 10.3164/jcbn.11005FR.

[22] Koriyama Y, Nakayama Y, Matsugo S, Kato S. Protective effect of lipoic acid against oxidative stress is mediated by Keap1/Nrf2-dependent heme oxygenase-1 induction in the RGC-5 cellline. Brain Res. 2013; 1499: 145-157. doi: 10.1016/j.brainres.2012.12.041.

[23] Park S, Karunakaran U, Jeoumg NH, Jeon JH, Lee IK. Physiological effect and therapeutic application of alpha lipoic acid. Curr Med Chem. 2014; 21 (32): 3636-3645. doi: $10.2174 / 0929867321666140706141806$.

[24] Hiller S, DeKroon R, Hamlett ED, Xu L, Osorio C, Robinette $\mathrm{J}$ et al. Alpha-lipoic acid supplementation protects enzymes from damage by nitrosative and oxidative stress. Biochim Biophys Acta. 2016; 1860 (1): 36-45. Doi: 10.1016/j.bbagen.2015.09.001.

[25] Serhiyenko V, Serhiyenko L, Suslik G, Serhiyenko A. Alphalipoic acid: mechanisms of action and beneficial effects in the prevention and treatment of diabetic complications. MOJ Public Health. 2018; 7 (4): 174-178. doi: 10.15406/mojph.2018.07.00224.

[26] Shay KP, Moreau RF, Smith EJ, Smith AR, Hagen TM. Alphalipoic acid as a dietary supplement: molecular mechanisms and therapeutic potential. Biochim Biophys Acta. 2009; 1790 (10): 1149-1160. doi: 10.1016/j.bbagen.2009.07.026.

[27] Ibrahimpasic K. Alpha lipoic acid and glycaemic control in diabetic neuropathies at type 2 diabetes treatment. Med Arch. 2013; 67 (1): 7-9. doi: 10.5455/medarh.2013.67.7-9.

[28] Morakinyo AO, Awobajo FO, Adegoke OA. Effects of alpha lipoic acid on blood lipids, renal indices, antioxidant enzymes, insulin and glucose level in streptozotocin-diabetic rats. Biol Med. 2013; 5: 26-33. http://www.biolmedonline.com. Accessed 20 Feb 2013.

[29] Feng B, Yan XF, Xue JL, Xu L, Wang H. The protective effects of $\alpha$-lipoic acid on kidneys in type 2 diabetic GotoKakisaki rats via reducing oxidative stress. Int J Mol Sci. 2013; 14 (4): 6746-6756. doi: 10.3390/ijms14046746. 
[30] Pitocco D, Tesauro M, Alessandro R, Ghirlanda G, Cardillo C. Oxidative stress in diabetes: implications for vascular and other complications. Int J Mol Sci. 2013; 14 (11): 2152521550. doi: 10.3390/ijms141121525.

[31] Sola S, Mir MQ, Cheema FA, Khan-Merchant N, Menon RG, Parthasarathy $\mathrm{S}$ et al. Irbesartan and lipoic acid improve endothelial function and reduce markers of inflammation in the metabolic syndrome: results of the Irbesartan and Lipoic Acid in Endothelial Dysfunction (ISLAND) study. Circulation.
2005; $\quad 111 \quad$ (3): $\quad 343-348 . \quad$ doi: 10.1161/01.CIR.0000153272.48711.B9.

[32] Serhiyenko V, Serhiyenko L, Krasnyi M, Serhiyenko A. Alpha-lipoic acid: therapeutic potential in diabetic neuropathies. Curre Res Diabetes Obes J. 2018; 7 (3): 555713. doi: 10.19080/CRDOJ.2018.07.555713. 